\title{
Advancements in Suppression of Osteosarcoma Tumorigenicity: A Prospective Look
}

\author{
Lin Wang ${ }^{1}$, Paul Park ${ }^{1}$, Frank La Marca ${ }^{1}$, Khoi Than ${ }^{1}$, Shayan Rahman ${ }^{1}$, Chia-Ying Lin ${ }^{1,2}$ \\ ${ }^{1}$ Spine Research Laboratory, Department of Neurosurgery, University of Michigan Medical School, Ann Arbor, USA; ${ }^{2}$ Department \\ of Biomedical Engineering, University of Michigan, Ann Arbor, USA. \\ Email: lincy@umich.edu
}

Received May 21 $1^{\text {st }}, 2012$; revised June $23^{\text {rd }}, 2012$; accepted July $3^{\text {rd }}, 2012$

\begin{abstract}
Bone morphogenetic proteins (BMPs) promote differentiation of stem cells into bone cells. Results from our pilot studies indicate these proteins are also capable of inducing the differentiation of stem-like cells that initiate and propagate osteosarcoma, a rare, highly malignant primary bone tumor affecting primarily children and adolescents. Our plans to evaluate the use of BMP as adjuvant therapy to suppress bone tumor while facilitating skeletal reconstruction are reviewed.
\end{abstract}

Keywords: BMP-2; Osteosarcoma; Cancer Stem Cells; Aldehyde Dehydrogenase

\section{Introduction}

Osteosarcoma (OSA) is an aggressive primary bone cancer primarily affecting children and adolescents, having significant impact on quality of life and survival. Classic OSA is a rare $(0.2 \%$ of all malignant tumors $)$ and highly malignant tumor, with an estimated incidence of 3 cases/ million population/year [1]. While surgical amputation of an affected limb may contribute to improved survival, patients suffer psychosocial and ambulatory consequences that can be particularly troubling to the adolescent population most affected by this disease [2]. Alternatively, regional resection of symptomatic bone tumors may be successfully performed if adequate stabilization of the structural support impaired by tumor resection can be achieved by bony fusion. Unfortunately, these patients also typically undergo radiation therapy, which negatively impacts bony fusion. Even without radiation, bony fusion can be difficult to achieve with standard fusion/ fixation techniques.

While the use of recombinant human bone morphogenetic proteins (BMPs) has been approved by the FDA to augment spinal fusion and recalcitrant long-bone nonunions, the use of BMP-2 is contraindicated in surgery for bone/spinal tumors due to concerns that this anabolic growth factor may contribute to tumor cell proliferation. Even though surgeons are eager to explore the feasibility of using BMP to facilitate skeletal reconstruction in bone tumor cases, studies to elucidate or support this application are relatively limited.
The inherent complexity of these tumors, especially in the context of the growth-factor-rich bone microenvironment, indicates the need for additional studies to allay this critical concern before clinical use of BMP for OSA treatment can be implemented. Recent evidence suggests the existence of cancer stem cells that are capable of reconstituting tumors hierarchically, postulating a new paradigm for the origin of cancer and its propagation [3-6]. Our early work also identified such tumor-initiating cells in OSA, which possess the abilities to form spherical colonies in an anti-adhesive environment, the plasticity to differentiate into multiple lineages, significant high-level expression of the 3 master genes Oct3/4A, Nanog, and Sox-2 that regulate stem cell characteristics, and an overwhelming propensity to reconstitute tumor subcutaneously and orthotopically (Figure 1), even with as few as 100 cells (Table 1) [7,8]. All of these characteristics have been referred to as stem cell attributes in cancer cells, and found to be highly correlated with high-level activity of aldehyde dehydrogenase (ALDH), an enzyme recently found to be highly active in stem and progenitor cells due to its link to retinoid metabolism that participates in terminal differentiation as well as self-renewal of certain cell types including various cancer stem cells [8-14].

Intriguingly, the results from our work indicate that BMP-2 is effective for inducing tumor-initiating cells associated with high levels of ALDH-namely ALDH ${ }^{\text {br }}$ cells-in the primary bone tumor, to express osteogenic phenotypes without stimulating proliferation, thereby drastically restricting tumor formation and expansion. 


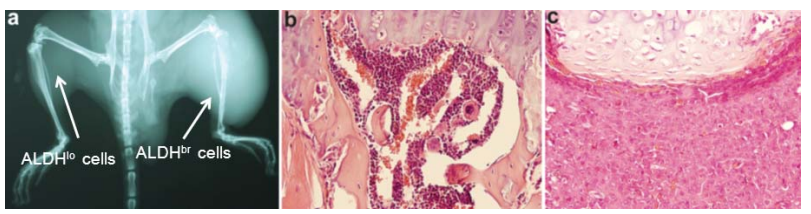

Figure 1. The astounding tumorigenicity of $\mathrm{ALDH}^{\mathrm{br}}$ cells was evident when these cells were inoculated at orthotopic sites. (a) When 10,000 ALDH ${ }^{\text {br }}$ cells were injected into tibiae of NOD/SCID mice, obvious osteolytic tumor lesions were identified radiographically at implantation site. In contrast, no tumors were noted in contra-lateral limbs injected with the same number of ALDH ${ }^{\text {lo }}$ cells. Histology sections documented that (b) intact bone structure was present at $\mathrm{ALDH}^{\mathrm{lo}}$ cell injection site, whereas (c) malignant cells were present in tumor formed by ALDH $^{\text {br }}$ cells.

Table 1. Tumor formation ability of $\mathbf{A L D H}^{\text {br }}$ cell population sorted from the primary OS99-1 xenograft.

\begin{tabular}{cccccc}
\hline Cell Type & $\begin{array}{c}10,000 \\
\text { Cells }\end{array}$ & $\begin{array}{c}5000 \\
\text { Cells }\end{array}$ & $\begin{array}{c}1000 \\
\text { Cells }\end{array}$ & $\begin{array}{c}500 \\
\text { Cells }\end{array}$ & $\begin{array}{c}100 \\
\text { Cells }\end{array}$ \\
\hline \multirow{2}{*}{$\mathrm{ALDH}^{\text {br }}$} & $3 / 3$ & $3 / 3$ & $3 / 3$ & $2 / 3$ & $2 / 3$ \\
& $(10 ;$ & $(13 ;$ & $(15 ;$ & $(16 ;$ & $(18 ;$ \\
$\mathrm{ALDH}^{\mathrm{lo}}$ & $0.3 \pm 1.3)^{*}$ & $10.6 \pm 1.3)^{*}$ & $12.6 \pm 1.9)^{*}$ & $14 \pm 2)^{*}$ & $14.5 \pm 3.5)^{*}$ \\
\hline
\end{tabular}

"Numbers in parentheses indicate: First, the time point (weeks) for the last grown tumor obtained within the same group; second, the average time period for tumor formation $\pm \mathrm{SD}$ (weeks) for the entire group.

Cell proliferation was significantly inhibited at 48 hours when $\mathrm{ALDH}^{\text {br }}$ cells were treated with $300 \mathrm{ng} / \mathrm{mL}$ BMP-2. Expression of key stem cell marker genes Oct3/4A, Nanog, and Sox-2 were all significantly lowered, and expression of phosphorylated Smad proteins 1, 5,8 were significantly elevated in BMP-2-treated $\mathrm{ALDH}^{\text {br }}$ cells compared to controls (data not shown). This was followed by upregulated expression of transcriptional factor RUNX2 that is essential for osteoblastic differentiation and skeletal morphogenesis, as well as type I collagen as an early marker of bone turnover, suggesting that BMP-2 may induce osteogenic differentiation of the targeted cells [15]. Subcutaneously, development of tumors formed by inoculated ALDH ${ }^{\text {br }}$ cells dramatically ceased when $30 \mu \mathrm{g}$ BMP-2 was administered at the lesion site in all experimental animals, with few Ki-67-positive cells present. Systemic as well as local skeletal consequences were also evaluated to ensure overall safety and efficacy of BMP-2. When human OSA cells were injected into the tibia medullary cavity of mice via intercondylar eminence to develop human OSA orthotopically, intact bone structure with no malignant cells was observed in histology sections of the limb injected with BMP-2, whereas malignant cells were dispersed over the entire intervened site along with fragmented bone matrix in the contra-lateral limb site injected with untreated tumor cells (Figures 2(a)-(c)). Osteogenesis induced by BMP-
2 in OSA seems to be a synergy from both $\mathrm{ALDH}^{\mathrm{br}}$ and $\mathrm{ALDH}^{\mathrm{lo}}$ subsets. When exposed to BMP-2, heterogenous $\mathrm{ALDH}^{\mathrm{lo}}$ progenies were induced to generate structured, calcified bone tissue in vivo with the morphological appearance of bone matrix deposition and marrow substances in the de novo bone tissue (Figures 2(d) and (e)), while tissue nodules of much smaller size were retrieved from the contra-lateral side where the same amount of $\mathrm{ALDH}^{\text {br }}$ cells were injected with BMP-2 (Figures 2(f) and (g)).

Using BMP-2 to inhibit tumorigenesis of ALDH ${ }^{\text {br }}$ cells thus appears to give great promise for suppressing or even autogenously eliminating bone tumors. We hypothesize that BMP-2 induction reduces the tumorigenic capacity of OSA while promoting osseous reunion of the intervened skeleton to facilitate surgical reconstruction after tumor resection. In particular, BMP-2 restricts the

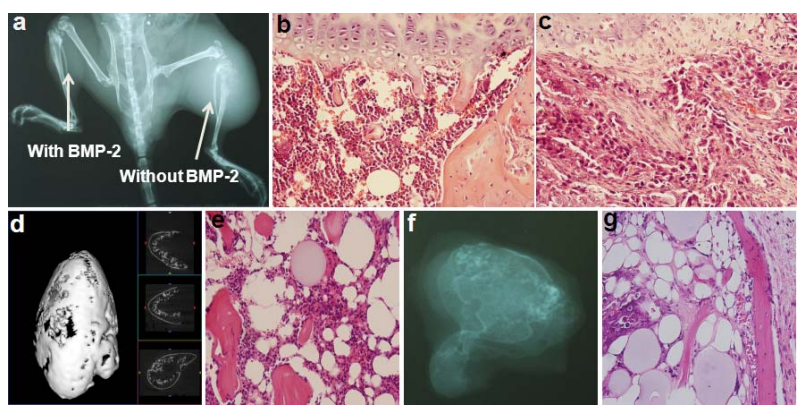

Figure 2. Suppression of OSA tumor growth by BMP-2. Unsorted human OSA-derived cells were introduced into the tibia medullary cavity via intercondylar eminence to develop human OSA orthotopically in mice as previously reported, either with or without concurrent BMP-2 application. (a) Twenty days after injection, bone tumor formation was noted with radiographic evidence of osteolysis at the site injected with untreated OSA cells. In contrast, no tumor was observed in the contralateral limb where cells were transplanted along with BMP-2; (b) Intact bone structure with no malignant cells was observed in histology sections of limb injected with BMP-2; whereas (c) malignant cells were dispersed over the entire intervened site along with fragmented bone matrix at the contra-lateral limb site injected with untreated tumor cells. Induced osteogenesis was contributed by both $A L D H^{\text {br }}$ cells and their ALDH $^{\text {lo }}$ progenies; (d) The 3-dimensionally rendered micro-CT illustrates that BMP-2 induced ALDH ${ }^{\text {lo }}$ cells to form well-structured bone nodule with an outer cortical shell containing inner cancellous bone; (e) Hematoxylin and eosin staining further confirmed the morphological appearance of bone matrix deposition and marrow substances in de novo bone tissue; (f, g) Tissue nodules of much smaller size were retrieved from the contra-lateral side where the same amount of $A L D H^{\text {br }}$ cells were injected with BMP-2, as also characterized radiographically and histologically. Cells were more differentiated and actively participated in bone tissue formation compared to those untreated ALDH ${ }^{\text {br }}$ cells in the earlier experiment that eventually appeared malignant. 


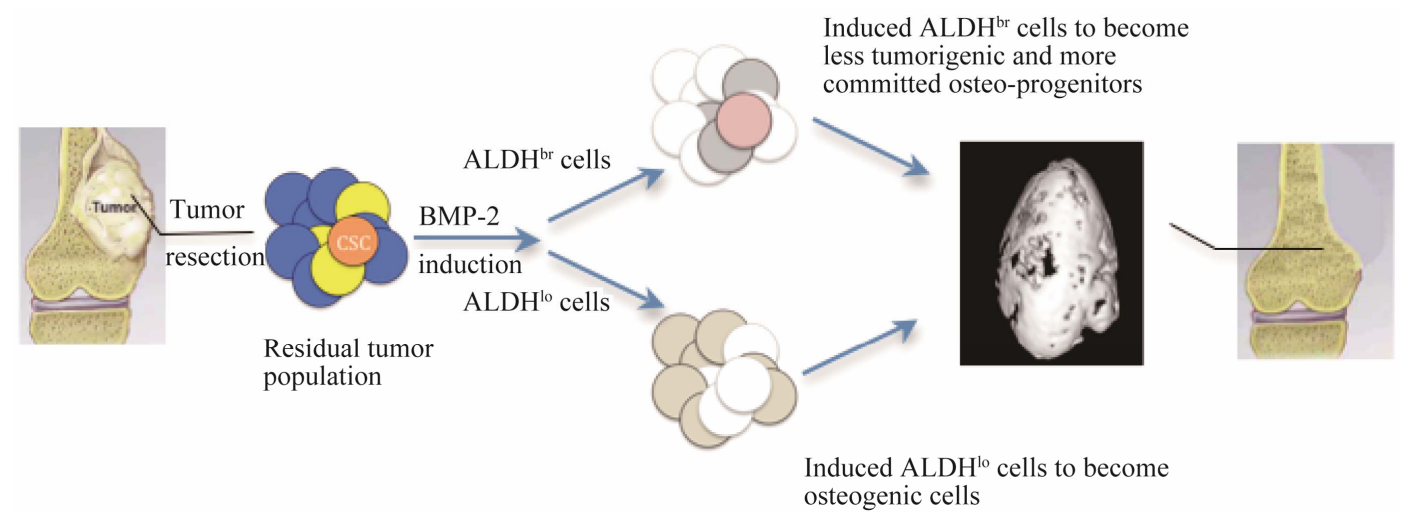

Figure 3. Schematic illustration of postulated hierarchical impact of BMP-2 in the heterogeneous tumor population of OSA.

tumorigenicity of OSA by inducing "differentiation" of the osteosarco-stem cells that express high levels of ALDH. Although our current work indicates that exposure to BMP-2 activates the Smad pathway in $\mathrm{ALDH}^{\text {br }}$ cells, which in turn promotes expression of certain key markers of osteogenic differentiation, more extensive characterizations will be conducted to investigate the final fate and behaviors of the induced cells. Moreover, we will use a canine spontaneous OSA model to evaluate if BMP treatment reduces tumor recurrence orthotopically while promoting stability of the intervened skeleton by induced bone augmentation as a preclinical investigation that replicates clinical scenarios.

We hope to demonstrate that the use of BMP as an adjuvant therapy to treat bone cancer would be of great benefit and provide sound support to facilitate development of a new strategy to augment limb-sparing treatments already in clinical use. Our concept of treating OSA with BMP-2, which is clinically available, not only meets the urgent need to seek potential therapeutics that are ready for application, but also uses the model cancer stem cell hypothesis to contribute new insight into hierarchical impacts to the heterogeneous population by the delivered cytokine, providing a new framework for treating OSA (Figure 3).

\section{Acknowledgements}

The authors gratefully acknowledge funding support provided by the National Institutes of Health. The project described was partially supported by Grant Number R01 AR056649 from NIAMS/NIH and the pilot research fund of the Department of Neurosurgery, University of Michigan. The authors would also like to thank Dr. Sheila M. Nielsen-Preiss from Montana State University for the generous gift of human osteosarcoma cell line OS99-1, Mrs. Holly Wagner for assistance in the preparation of the manuscript, and Mr. Martin J. White for flow cytometry.

\section{REFERENCES}

[1] P. Picci, "Osteosarcoma (Osteogenic Sarcoma)," Orphanet Journal of Rare Diseases, Vol. 2, 2007, p. 6. doi:10.1186/1750-1172-2-6

[2] T. A. Damron, W. G. Ward and A. Stewart, "Osteosarcoma, Chondrosarcoma, and Ewing's Sarcoma: National Cancer Data Base Report," Clinical Orthopaedics and Related Research, Vol. 459, 2007, pp. 40-47. doi:10.1097/BLO.0b013e318059b8c9

[3] D. Bonnet and J. E. Dick, "Human Acute Myeloid Leukemia Is Organized as a Hierarchy that Originates from a Primitive Hematopoietic Cell," Nature Medicine, Vol. 3, 1997, pp. 730-737. doi:10.1038/nm0797-730

[4] D. Hanahan and R. A. Weinberg, "The Hallmarks of Cancer," Cell, Vol. 100, No. 1, 2000, pp. 57-70. doi:10.1016/S0092-8674(00)81683-9

[5] T. Reya, S. J. Morrison, M. F. Clarke and I. L. Weissman, "Stem Cells, Cancer, and Cancer Stem Cells," Nature, Vol. 414, No. 6859, 2001, pp. 105-111. doi: $10.1038 / 35102167$

[6] B. Vogelstein and K. W. Kinzler, "Cancer Genes and the Pathways They Control," Natural Medicine, Vol. 10, No. 8, 2004, pp. 789-799. doi:10.1038/nm1087

[7] L. Wang, P. Park and C. Y. Lin, "Characterization of Stem Cell Attributes in Human Osteosarcoma Cell Lines," Cancer Biology \& Therapy, Vol. 8, No. 6, 2009, pp. 543552. doi:10.4161/cbt.8.6.7695

[8] L. Wang, P. Park, H. Zhang, F. La Marca and C. Y. Lin, "Prospective Identification of Tumorigenic Osteosarcoma Cancer Stem Cells in OS99-1 Cells Based on High Aldehyde Dehydrogenase Activity," International Journal of Cancer, Vol. 128, No. 2, 2011, pp. 294-303. doi:10.1002/ijc. 25331

[9] A. M. Cheung, T. S. Wan, J. C. Leung, L.Y. Chan, H. Huang, Y. L. Kwong, R. Liang and A. Y. Leung, "Aldehyde Dehydrogenase Activity in Leukemic Blasts Defines a Subgroup of Acute Myeloid Leukemia with Adverse Prognosis and Superior NOD/SCID Engrafting Potential," Leukemia, Vol. 21, 2007, pp. 1423-1430. doi:10.1038/sj.leu.2404721

[10] C. Ginestier, M. H. Hur, E. Charafe-Jauffret, F. Monville, J. Dutcher, M. Brown, J. Jacquemier, P. Viens, C. G. 
Kleer, S. Liu, A. Schott, D. Hayes, D. Birnbaum, M. S. Wicha and G. Dontu, "ALDH1 Is a Marker of Normal and Malignant Human Mammary Stem Cells and a Predictor of Poor Clinical Outcome," Cell Stem Cell, Vol. 1, No. 5, 2007, pp. 555-567. doi:10.1016/j.stem.2007.08.014

[11] E. H. Huang, M. J. Hynes, T. Zhang, C. Ginestier, G. Dontu, H. Appelman, J. Z. Fields, M. S. Wicha, B. M. Boman, "Aldehyde Dehydrogenase 1 Is a Marker for Normal and Malignant Human Colonic Stem Cells (SC) and Tracks SC Overpopulation during Colon Tumorigenesis," The Journal of Cancer Research, Vol. 69, 2009, pp. 3382-3389. doi:10.1158/0008-5472.CAN-08-4418

[12] M. B. Kastan, E. Schlaffer, J. E. Russo, O. M. Colvin, C. I. Civin and J. Hilton, "Direct Demonstration of Elevated Aldehyde Dehydrogenase in Human Hematopoietic Progenitor Cells," Blood, Vol. 75, No. 10, 1990, pp. 19471950.

[13] S. Ma, K. W. Chan, T. K. Lee, K. H. Tang, J. Y. Wo, B. J.
Zheng and X. Y. Guan, "Aldehyde Dehydrogenase Discriminates the CD133 Liver Cancer Stem Cell Populations," Molecular Cancer Research, Vol. 6, 2008, pp. 11461153. doi:10.1158/1541-7786.MCR-08-0035

[14] N. E. Sladek, R. Kollander, L. Sreerama and D. T. Kiang, "Cellular Levels of Aldehyde Dehydrogenases (ALDH1A1 and ALDH3A1) as Predictors of Therapeutic Responses to Cyclophosphamide-Based Chemotherapy of Breast Cancer: A Retrospective Study. Rational Individualization of Oxazaphosphorine-Based Cancer Chemotherapeutic Regimens," Cancer Chemotherapy and Pharmacology, Vol. 49, No. 4, 2002, pp. 309-321. doi:10.1007/s00280-001-0412-4

[15] L. Wang, P. Park, H. Zhang, F. La Marca, A. Claeson, J. Valdivia and C. Y. Lin, "BMP-2 Inhibits the Tumorigenicity of Cancer Stem Cells in Human Osteosarcoma OS99-1 Cell Line," Cancer Biology Therapy, Vol. 11, No. 5, 2011 pp. 457-463. doi:10.4161/cbt.11.5.14372 